\title{
Modeling Underwater Acoustic Communications for Multi-Robot Missions in a Robotics Simulator
}

\author{
Anuj Sehgal, Daniel Cernea, Andreas Birk \\ Computer Science \\ Jacobs University Bremen \\ Campus Ring 1, 28759 Bremen, Germany \\ \{s.anuj, d.cernea, a.birk\}@jacobs-university.de
}

\begin{abstract}
Missions involving multiple Autonomous Underwater Vehicles (AUVs) are gaining increasing popularity with the advent of better control mechanisms and availability of acoustic modems that allow for cooperative networked tasks to be carried out by Unmanned Underwater Vehicles (UUVs). However, high costs associated with offshore testing and the lack of useful tools to accurately simulate multi-AUV tasks has been a hindrance to exploiting the full potential of the field. Even though there exist a few simulators for modeling a single AUV, there is virtually no simulation tool available that allows modeling of acoustically networked communications between multiple AUVs by accurately characterizing the underwater acoustic channel. In this paper we present an overview on modeling of the underwater acoustic channel, taking into account the high degree of local variability of ocean conditions, multi-path echoes and ambient noise, within the framework of an underwater acoustic communications server for the Unified System for Automation and Robotics Simulator (USARSim) robotics simulator.
\end{abstract}

\section{INTRODUCTION}

Autonomous Underwater Vehicles (AUVs) have been steadily gaining increased acceptance for deployments in offshore research and exploration tasks. However, as in the terrestrial and aerial robotics fields, the maximum potential for underwater unmanned vehicles lies in cooperative multiAUV tasks or those involving AUV and diver coordination. The effective design and testing of cooperation algorithms has thus far been limited and difficult due to the relatively high cost of off-shore missions. Since the radio channel does not function underwater and the optical channel has very limited transmission range [1], most wireless underwater communications between Unmanned Underwater Vehicles (UUVs) are implemented using the underwater acoustic channel.

Though these acoustic networks enable the use of wireless networks in a host of applications for the underwater environment, the acoustic channel access method also poses some very important challenges to achieving near real-time communications in the form of limited bandwidth-capacity, low battery power availability with none to little possibility of recharging and the high likelihood of network disruptions [2]. While tackling the aforementioned problems any underwater acoustic system must also take into account issues such as long and varying propagation delays, multi-path echoes and high and varying ambient noise. As such, the overall acoustic communication design process is error-prone due to the high number of variables in the system.
From fabrication to deployment the costs associated with multiple AUVs organized in a network can be quite high. The costs of a dependable underwater acoustic modem are in the order of several thousand dollars and off shore deployment and recovery of underwater vehicles from a small boat can be in thousands per day. Such high costs associated with offshore testing can be a bane to development in case revisions are necessary [3]. These costs coupled with complexity of the channel highlight the need for a simulator to accurately model not only the effects of the underwater environment on UUVs, but also the rapidly changing channel which is used for communication between UUVs. This would assist in development of systems and reduce off-shore deployment times.

Even though there are plenty of robotics simulators available [4], not much work has been put into simulating the underwater environment and even lesser work has concentrated on simulating network communication between AUVs [5]. The popular Unified System for Automation and Robotics Simulator (USARSim) has built in support for simulating UUVs. This combined with the large user community behind USARSim, a strong physics engine and its proven capabilities in simulating multi-robot cooperative tasks makes it an ideal simulation environment for multi-AUV missions and communications. The ocean being a highly complex medium for the propagation of sound, due to inhomogeneities and random fluctuations, including effects of the rough seas and ocean bottom variances, acoustics communication channel models that would provide feedback on communications were also implemented within the Wireless Simulation Server (WSS) plugin for USARSim.

The following sections provide an insight on acoustic propagation models which were developed along with information on the channel characterization approach that was used for the simulator. This is followed by an overview on the USARSim robotics simulator and the extensions we made to it in order to enable simulations of mobile underwater vehicles. A discussion on the extensions to WSS for enabling underwater simulations and also some results based on our test cases is followed by conclusions.

\section{Acoustic Propagation Model}

Even though wireless connectivity is achievable underwater by using the acoustic medium, the acoustic channel is considerably different from the commonly used radio channel [6], 
thereby indicating the need for a complete channel model that can be used in simulation work. In this section we present the different aspects of the underwater acoustic channel as they were used in our simulation model.

\section{A. Propagation Delay}

For most purposes the speed of sound in water is taken to be approximately $1500 \mathrm{~m} / \mathrm{s}$. While this is accurate within a certain range, the underwater channel is a complex environment which is effected by many varying factors, primarily temperature, salinity and depth. Furthermore each of these factors may also be interdependent or varying across the ocean. It is, as such, important to have an accurate model of the effects of these parameters on the speed of sound in water.

The speed of sound in water has been a focus of analysis by many mathematical models. We chose to utilize the expression proposed by the authors of [7] since it calculates the speed of sound in water with an error in the speed estimate in the range of approximately $0.070 \mathrm{~m} / \mathrm{s}$ :

$$
\begin{aligned}
v= & 1448.96+4.591 C-5.304 \cdot 10^{-4} C^{3} \\
& +1.340(S-35)+1.630 \cdot 10^{-2} D+1.675 \cdot 10^{-7} D^{2} \\
& -1.025 \cdot 10^{-2} C(S-35)-7.139 \cdot 10^{-13} C D^{3}
\end{aligned}
$$

where $v$ is the sound velocity in $\mathrm{m} / \mathrm{s}, C$ is the temperature in degrees Celsius, $S$ is the salinity in parts per trillion (ppt) and $D$ is the depth in meters.

Since the speed of sound can vary greatly in regions of thermocline and halocline, and most AUV missions operate within these regions [8], determining speed of sound accurately is crucial.

\section{B. Propagation Loss}

Table I

VALUES FOR REPRESENTING TYPES OF GEOMETRICAL SPREADING VIA THE GEOMETRICAL SPREADING COEFFICIENT $k$

\begin{tabular}{|c|c|c|c|}
\hline & Spherical & Cylindrical & Practical \\
\hline \hline$k$ & 2 & 1 & 1.5 \\
\hline
\end{tabular}

Propagation loss is composed mainly of three aspects, namely, geometrical spreading, attenuation by absorption and the anomaly of propagation. The latter is nearly impossible to model and as such the attenuation, in $\mathrm{dB}$, that occurs over a transmission range $l$ for a signal frequency $f$ can be obtained by:

$$
10 \log A(l, f)=k \cdot 10 \log l+l \cdot \alpha
$$

where $\alpha$ is the absorption coefficient in $\mathrm{dB} / \mathrm{km}$ and $k$ represents the geometrical spreading factor. Geometrical spreading loss can be widely categorized as spherical or cylindrical. Cylindrical spreading occurs when the transmitter and receiver are located a short distance, while spherical spreading is pronounced in long range communications. The geometrical spreading factor can be substituted with values shown in Table I in order to represent the type of spreading that occurs.

\section{Absorption Coefficient}

Attenuation by absorption occurs due to the conversion of acoustic energy within seawater into heat. This process of attenuation is frequency dependent since at higher frequencies more energy is absorbed. There are several equations describing the processes of acoustic absorption in seawater which have laid the foundation for current knowledge.

Table II

FISHER \& SIMMONS' MODEL COEFFICIENTS

$$
\begin{aligned}
& A_{1}=1.03 \times 10^{-8}+2.36 \times 10^{-10} \cdot T-5.22 \times 10^{-12} \cdot T^{2} \\
& A_{2}=5.62 \times 10^{-8}+7.52 \times 10^{-10} \cdot T \\
& A_{3}=\left[55.9-2.37 \cdot T+4.77 \times 10^{-2} \cdot T^{2}-3.48 \times 10^{-4} \cdot T^{3}\right] \cdot 10^{-15} \\
& f_{1}=1.32 \times 10^{3}(T+273.1) e^{\frac{-1700}{T+273.1}} \\
& f_{2}=1.55 \times 10^{7}(T+273.1) \mathrm{e}^{\frac{-3052}{T+273.1}} \\
& P_{1}=1 \\
& P_{2}=1-10.3 \times 10^{-4} \cdot P+3.7 \times 10^{-7} \cdot P^{2} \\
& P_{3}=1-3.84 \times 10^{-4} \cdot P+7.57 \times 10^{-8} \cdot P^{2}
\end{aligned}
$$

The attenuation by absorption is dependent upon the ambient conditions, transmission frequency and distance, and as such, the Fisher \& Simmons model proposed in [9] is used for the modeling work presented here. This model also takes into account the effects of relaxation frequencies caused by the presence of boric acid and magnesium sulphate in the ocean.

In Equation $3 A_{1}, A_{2}$ and $A_{3}$ represent the effects of temperature on signal absorption, while $P_{1}, P_{2}$ and $P_{3}$ represent the effects of depth and $f_{1}$ and $f_{2}$ represent the relaxation frequencies introduced due to the absorption caused by the presence of boric acid and magnesium sulphate in oceanic water. These coefficients may be obtained from Table II.

$$
\alpha=A_{1} P_{1} \frac{f_{1} f^{2}}{f_{1}^{2}+f^{2}}+A_{2} P_{2} \frac{f_{2} f^{2}}{f_{2}^{2}+f^{2}}+A_{3} P_{3} f^{2}
$$

\section{Ambient Noise Model}

Table III

Formulae Providing PSD of the Ambient Noise

$$
\begin{gathered}
10 \log N_{t}(f)=17-30 \log f \\
10 \log N_{s}(f)=40+20(s-0.5)+26 \log f-60 \log (f+0.03) \\
10 \log N_{w}(f)=50+7.5 w^{\frac{1}{2}}+20 \log f-40 \log (f+0.4) \\
10 \log N_{t h}(f)=-15+20 \log f
\end{gathered}
$$

Ambient noise in the ocean can be described as Gaussian and having a continuous power spectral density (PSD). The four most prominent sources for ambient noise are the turbulence, shipping, wind driven waves and thermal noise. The PSD in $\mathrm{dB}$ re $\mu \mathrm{Pa}$ per $\mathrm{Hz}$ for each of these is given by the formulae [10] shown in Table III.

The ambient noise in the ocean is colored and hence different factors have pronounced effects in specific frequency 
ranges. In the noise model equations we utilize, this colored effect of noise is represented by $N_{t}$ as the turbulence noise, $N_{s}$ as the shipping noise ( $s$ as the shipping factor lies between 0 and 1), $N_{w}$ as the wind driven wave noise ( $w$ as the wind speed in $m / s$ ) and $N_{t h}$ as the thermal noise. The overall noise PSD may be obtained from:

$$
N(f)=N_{t}(f)+N_{s}(f)+N_{w}(f)+N_{t h}(f)
$$

\section{Channel Characterization Model}

For the purpose of simulation the performance of the underwater acoustic channel can be characterized by received signal power, signal-to-noise ratio (SNR) and the capacity. We utilize the equations presented by the authors of [6], [11] in order to perform the channel characterization in our simulator.

\section{A. Received Signal Power}

If a signal with frequency $f$ is transmitted over distance $l$ with a power $P_{t x}$ then we can calculate the arriving signal power $P_{r x}$ in $\mathrm{dB}$ :

$$
10 \log P_{r x}=10 \log P_{t x}-10 \log A(l, f)
$$

The result obtained from Equation 5 takes only the case for directional transmission into account, i.e., the most direct propagation path from transmitter to receiver. However, in case a transmission that is not directional needs to be modeled, this equation can be extended for the indirect routes as well.

\section{B. Signal-to-noise Ratio}

The signal-to-noise ratio (SNR) of an emitted underwater acoustic signal at the receiver can be expressed by the passive sonar equation [10]:

$$
S N R=S L-T L-N L+D I
$$

Here, $S L$ is the source level, $T L$ is the transmission loss, $N L$ is the noise level and $D I$ is the directivity index. The path loss represented by Equation 2 is the transmission loss $T L$. The noise level $N L$ may easily be obtained from Equation 4 , leading to the rewriting of the passive sonar equation for obtaining the SNR, when a particular transmission frequency $f$ is used over a certain distance $l$, in $\mathrm{dB}$ :

$$
\begin{aligned}
10 \log S N R(l, f)= & 10 \log P_{t x}-10 \log A(l, f) \\
& -10 \log N(f)-10 \log B
\end{aligned}
$$

where $B$ is the modem bandwidth.

Rewriting Equation 7 can be useful in determining the transmission power necessary in order to maintain a minimum SNR for a certain transmission distance and frequency at a particular depth and ambient oceanic conditions.

\section{Bandwidth and Channel Capacity}

Most current reported results focus on the channel capacity calculations that do not take into account the effect of parameters based on the ambient ocean environment and network deployment topology. The authors of [12] use a channel model with additive Gaussian noise and in [13] the work focuses on using a Rayleigh fading model along with additive white Gaussian noise. The work described in [6], [14] establishes the relationship between capacity and distance, while the authors of [11] show the dependence of capacity on depth and temperature as well.

As such, in order to account for the dependency of capacity on transmission frequency and distance, depth and temperature, we utilize equations proposed by [11]. Bandwidth, $B$, is dictated by the modem choice. We utilize the Shannon theorem to determine the maximum capacity bound and by extrapolating from Equation 7 we obtain the channel capacity, $\mathrm{C}$, over a transmitted distance $l, C(l)$ by:

$$
C(l)=\int_{B} \log _{2}\left(1+\frac{P_{t x}}{A(l, f) N(f) B}\right) d f
$$

\section{USARSIM AND UNDERWATER SIMULATIONS}

USARSim is a high-fidelity simulation tool for simulating robots and environments based on the Unreal Tournament game engine. USARSim is the basis for important currentday robotic simulations, the most famous being represented by the RoboCup rescue virtual robot competition. USARSim provides superior visual rendering and physical modeling due to an underlying physics engine. This enables the entire effort to be devoted to the robotics-specific tasks of modeling platforms, control systems, sensors, interface tools and environments. Advanced editing features for almost every aspect of the simulation, with a special focus on robots and environments, further adds to the advantages of USARSim.

All these advantages and its modular nature in developing new additions for sensors, modules and ability to model complex underwater environments on the strengths of a proven physics engine makes it a suitable tool to model the multiAUV underwater acoustic communications in as well. In this section we provide some details on the underwater environmental and submersible vehicle modeling capabilities of USARSim along with information on WSS and the extensions we made to both these tools in order to enable mobile multiAUV communication simulations.

\section{A. Underwater Environments}

In order to correctly evaluate the communication model and test the effects of algorithms, methods and control schemes, it is important to have environment and robot models that mimic reality. USARSim has a model world simulating an underwater environment available by default, but others can also be easily created using the Unreal Tournament model editor. We used the default model containing water as our testing world model. 


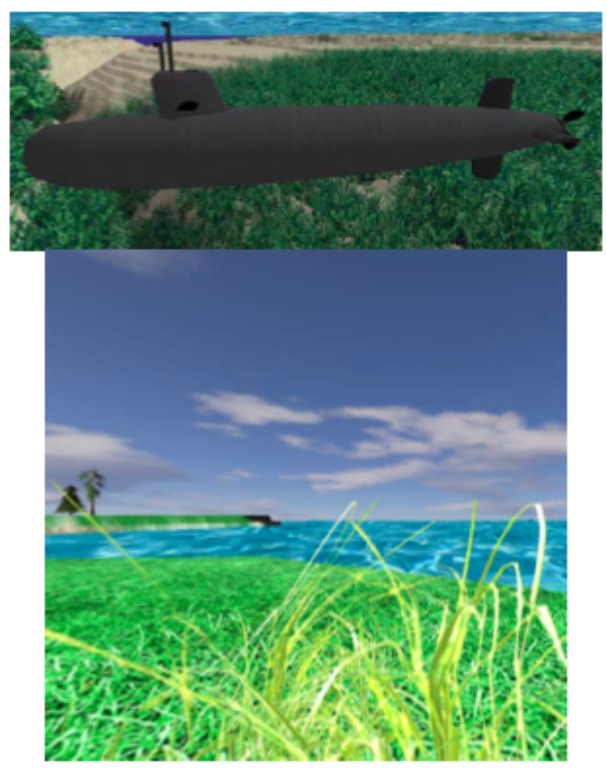

Figure 1. Screenshot of the USARSim default model and submarine

\section{B. Vehicle Model}

Though any vehicle models can be created and imported into the USARSim environment, we chose to use the Submarine model which is provided by default. This model can have sonar sensors, imaging sensors, echo-sounders, side scan and an optical camera simulated on it. The default implementation of the library to interface with USARSim did not have an implementation of a driving mechanism for the submarine and as such we implemented a drive mechanism for the propeller, rudder and stern planes, thereby providing us full mobility control of the submarine and giving us access to testing mobile-AUV communications.

\section{Wireless Simulation Server}

WSS is an USARSim plugin that enables simulation of $802.11 \mathrm{x}$ wireless network links. WSS works using plugins to implement propagation models allowing for further extensibility. The signal degradation is calculated based upon parameters that are setup for the propagation model plugin and it governs whether connection between robots is possible or not.

Our channel model was implemented as a propagation model plugin for WSS. The model configuration dialog in Figure 3 shows how the user can configure the ambient noise parameters to suit the real environment being modeled. Since USARSim does not have a way to provide the depth of the robot to WSS, a sea level function was implemented. This defines the sea level in the world map so that the robot's depth could be calculated using its Cartesian coordinates. The determined depth is used to obtain the temperature from the global thermocline average to compute the propagation delay and attenuation coefficient.

The user can also specify the signal transmission strength, cutoff strength, bandwidth and center frequency to model any modem without making changes to WSS or USARSim.

WSS by default only supports robots being able to retrieve signal strength for the target robot. This is inadequate for the

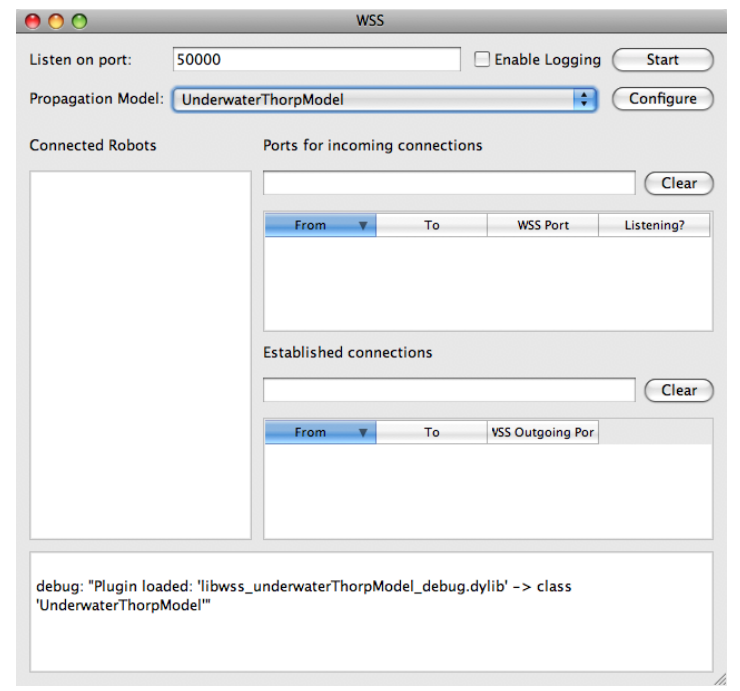

Figure 2. Screenshot of the USARSim WSS capable of simulating underwater networks

\begin{tabular}{|c|c|c|c|}
\hline \multicolumn{4}{|c|}{ UnderwaterThorpModel Configuration } \\
\hline \multicolumn{4}{|l|}{ USARSim Connection } \\
\hline \multicolumn{2}{|l|}{ Host $127 \cdot 0.0 .1$} & 7435 & $:$ \\
\hline \multicolumn{4}{|c|}{ Underwater Noise Parameters } \\
\hline Shipping 1.000 & $\because$ Wind Speed & 0.000 & $\theta$ \\
\hline Spreading 1.50 & - Sea Level & 16.00 & $\theta$ \\
\hline \multicolumn{4}{|l|}{ Modem Characteristics } \\
\hline Signal Transmit & \multicolumn{2}{|c|}{$190.000 \mathrm{~dB}$} & $\Leftrightarrow$ \\
\hline Signal Cutoff & \multicolumn{2}{|l|}{$150.000 \mathrm{~dB}$} & $\theta$ \\
\hline Frequency & \multicolumn{2}{|l|}{$30.00 \mathrm{kHz}$} & 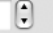 \\
\hline \multirow[t]{2}{*}{ Bandwidth } & \multicolumn{2}{|l|}{$1.500 \mathrm{kHz}$} & 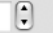 \\
\hline & \multicolumn{3}{|c|}{ Cancel $\bigcirc \mathrm{OK}$} \\
\hline
\end{tabular}

Figure 3. Screenshot of the propagation model configuration window

underwater networking scenario, where the ability to retrieve propagation delay and channel capacity is also important. As such, we extended WSS to support the following functions:

- GETPD returns the propagation delay between the querying robot and the target robot specified in the query string.

- GETBW returns the channel capacity in kbps between the querying robot and the target robot specified in the query string.

A major advantage of the USARSim simulation environment is that of being able to successfully emulate an underwater environment. In other words, the environmental modeling ability gives the capability of also modeling and obtaining a surface-bottom profile of the ocean floor. This is helpful since the surface-bottom of the ocean is a great contributor to signal interference as a result of reflections that occur from the seabed in shallow water acoustic communications. Furthermore, the surface-bottom profile can have a significant effect upon multipath propagation interference as well. As such, it is important to be able to test the likelihood of this factor interfering with the transmission signal. In order to implement the ability to 
retrieve surface-bottom multi-path signal interference WSS was extended to support the following function as well:

- GETML returns the interference likelihood as 0 or 1 for a distance to the surface-bottom provided in the query string.

In order to use the GETML function the bottom profile between the transmitting and receiving AUV is retrieved as a depth map by performing a ray trace at multiple points towards the surface-bottom of the map along a straight line between the transmitter and receiver. Another ray trace between the transmitter, surface-bottom points and receiver is performed in order to obtain the path that a transmitted signal would take, when it is reflected off the surface-bottom. The total distance travelled by a reflected wave, from transmitter to surfacebottom and then to receiver, is used in order to determine the arriving signal strength on a particular path. In case, the PSD of any of the sampled paths is higher than the minimum required reception strength, as determined by the modem properties, multi-path signal interference is determined to be likely.

WSS performs the channel characterization in real-time, as the simulation is executed within USARSim and provides feedback to the querying robots. This real-time calculation allows the simulation to take into account any changes that may occur in the environment or any actions the submersible vehicle might be taking at the time.

\section{Simulation Results}

The USARSim simulator provides a familiar environment to develop and perform multi-AUV simulations in. However, before the results of a simulator can be considered dependable, they must be validated. As such, this section provides an overview on the results obtained from the simulator and compares them to previously reported results.

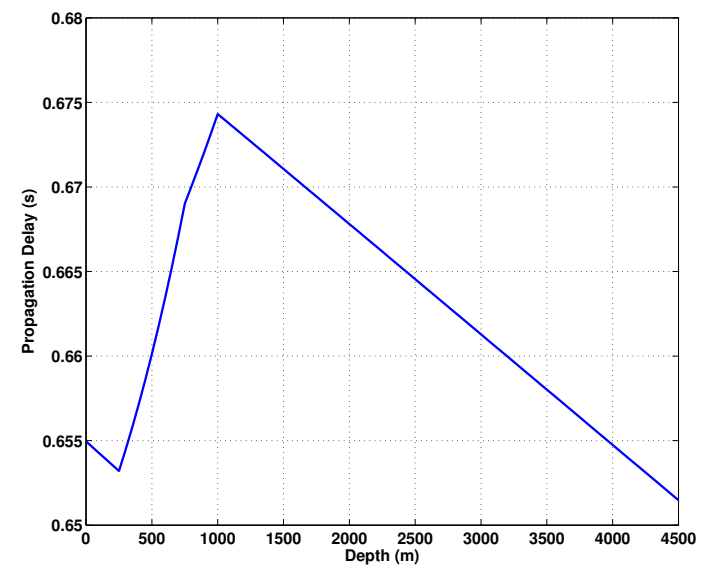

Figure 4. The change in propagation delay with depth of the two nodes. The propagation delay curve follows a shape similar to that of the sound velocity profile.

In order to predict the accuracy of the propagation delay calculated by the simulator, an experiment similar to the one run by Harris et al. [14] was executed with two nodes, both situated $1 \mathrm{~km}$ apart. The depth of both these nodes was progressively increased while maintaining the same depth for both the nodes and keeping the $1 \mathrm{~km}$ distance between them constant. The resulting values of propagation delay are plotted in Figure 4.

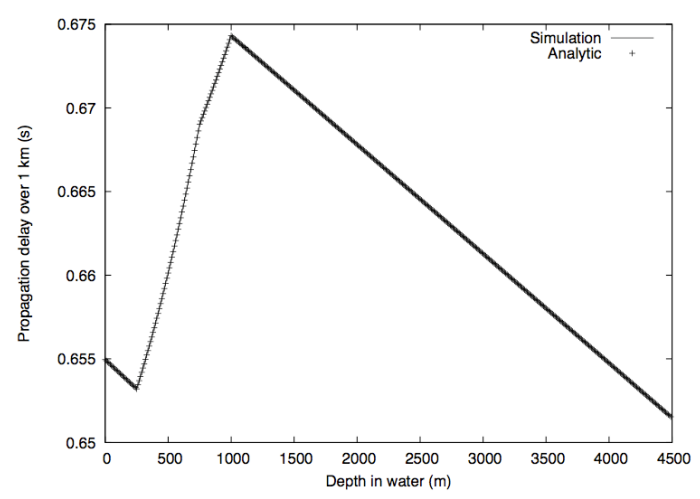

Figure 5. Propagation Delay as obtained by the simulative and analytical study conducted by Harris et al. [14].

A comparison between the results obtained and previously published literature can be obtained from Figures 4 and 5. It is evident that USARSim WSS results mimic those previously reported.

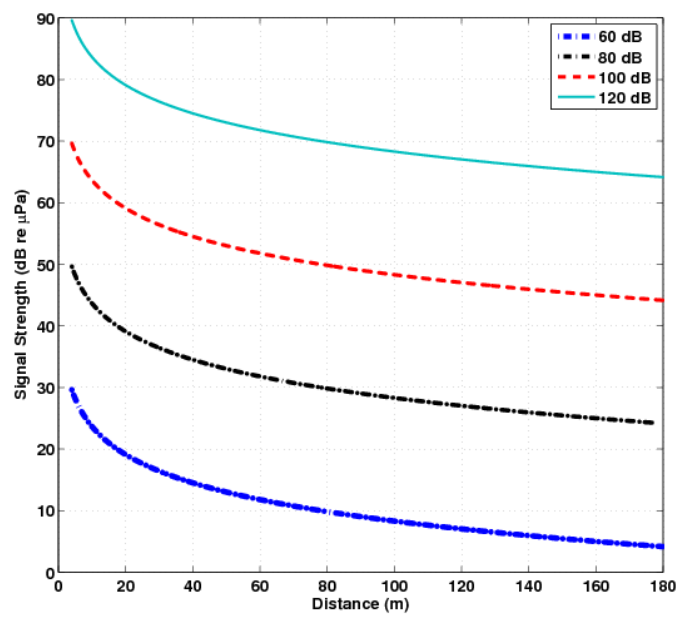

Figure 6. The arriving signal strength while the distance between the transmitting and receiving nodes was varied between 4 to $180 \mathrm{~m}$ and the transmit power is also changed.

The arriving signal strength is very useful in determining the quality of the arriving signal. The evaluation of the arriving signal strength is not a straightforward comparison like other values since it is dependent upon the transmission signal strength and the work in published literature is based upon the transmission strength necessary to achieve a desired SNR. As such, in order to test the accuracy of the simulator, it is important to draw a few inferences from available data.

It is known that available capacity drops with distance and to achieve a higher capacity higher transmission power is necessary [11] . Conversely, available capacity is proportional 
to the transmission power utilized. As such, we can deduce that the signal strength should reduce with distance in a somewhat logarithmic fashion.

Keeping this in mind an experiment while keeping a depth of $100 \mathrm{~m}$ constant, using the standard thermocline and varying the distance between the two nodes between $4 \mathrm{~m}$ and $180 \mathrm{~m}$ and also changing the transmission power between $60 \mathrm{~dB}$ and $120 \mathrm{~dB}$, was executed in the USARSim WSS environment. The results of this experiment can be seen in Figure 6. Since the shape of this figure follows the expected shape, it can be deduced that the simulator works accurately.

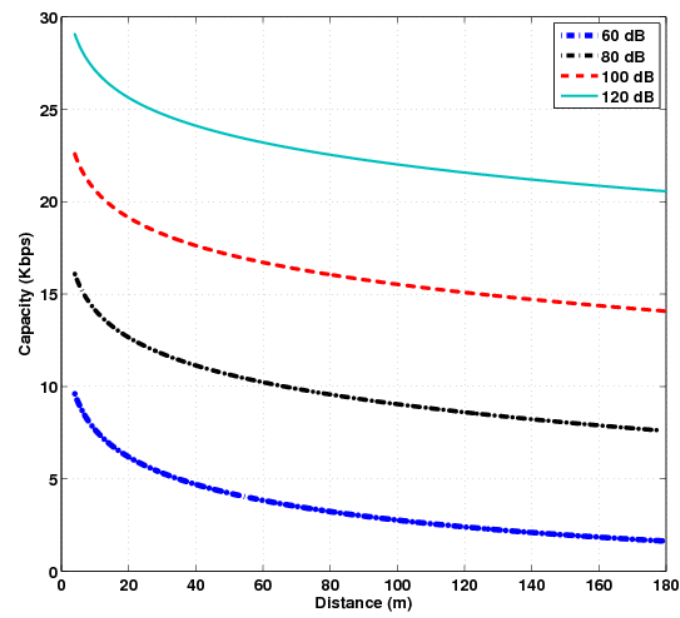

Figure 7. The channel capacity while the distance between the transmitting and receiving nodes was varied between 4 to $180 \mathrm{~m}$ and the transmit power is also changed.

Using the same experiment as the one which provided results for received signal power, the values of channel capacity were also obtained and plotted in Figure 7.

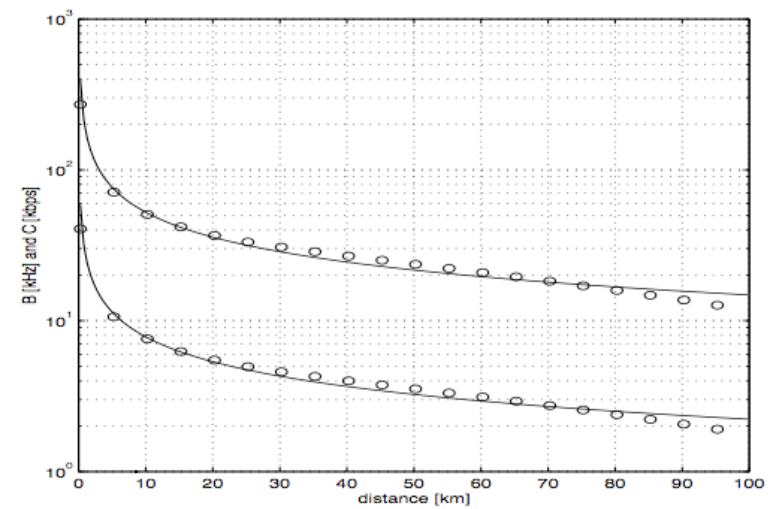

Figure 8. The bandwidth and capacity while the distance between the transmitting and receiving nodes was varied during the study conducted by Stojanovic et al. [6] (Upper line is capacity).

It is clear from Figure 7 that the capacity reduces with distance between the nodes and increases with increased transmission power. Upon comparing obtained results to Stojanovic et al's [6], depicted in Figure 8, it is clear that the shape of the curves is very similar irrespective of the transmission frequency and power utilized. The similarity in the shape of the curves argues in the favor of the overall robustness of results provided by the simulators.

\section{CONCLUSIONS}

In this work we have implemented propagation models for the underwater acoustic channel within the framework of the USARSim robotics simulator by extending the WSS plugin. Our models are based upon accepted theoretical models and provide results which are as close to off-shore performance as possible. Furthermore, using a simulator with a realistic physics engine ensures further accuracy. The computation of the multi-path interference likelihood based upon sub-bottom profile is unique to our implementation and provides a feature that could help researchers build systems which minimize effects of this phenomenon.

The results obtained from the simulator and their comparison to published results validates the capabilities of our implementation. With the implementation of underwater acoustic channel models, the USARSim-WSS combination provides an effective modeling and simulation tool for many fields, including for the underwater robotics and communication research.

\section{REFERENCES}

[1] I. F. Akyildiz, D. Pompili, and T. Melodia, "Challenges for efficient communication in underwater acoustic sensor networks," SIGBED Rev., vol. 1, no. 2, pp. 3-8, 2004.

[2] E. Sozer, M. Stojanovic, and J. Proakis, "Underwater acoustic networks," IEEE Journal of Oceanic Engineering, vol. 25, pp. 72-83, January 2000.

[3] J. Partan, J. Kurose, and B. N. Levine, "A survey of practical issues in underwater networks," in WUWNet '06: Proceedings of the 1st ACM international workshop on underwater networks, (New York, NY, USA), pp. 17-24, ACM, 2006.

[4] J. Craighead, R. Murphy, J. Burke, and B. Goldiez, "A survey of commercial \& open source unmanned vehicle simulators," in 2007 IEEE International Conference on Robotics and Automation, 2007.

[5] T. Bielohlawek, "Subsim - an autonomous underwater vehicle simulation system," Master's thesis, Universitaet Kaiserslautern, 2006.

[6] M. Stojanovic, "On the relationship between capacity and distance in an underwater acoustic communication channel,' in WUWNet '06. Proceedings of the 1st ACM international workshop on underwater networks, (New York, NY, USA), pp. 41-47, ACM, 2006.

[7] K. V. MacKenzie, "Nine-term equation for sound speed in the oceans," Acoustical Society of America Journal, vol. 70, pp. 807-812, Sept. 1981.

[8] A. Caiti, E. Crisostomi, and A. Munafo, "Physical characterization of acoustic communication channel properties in underwater mobile sensor networks," in Proceedings of International Conference on Sensor Systems and Software, (Pisa, Italy), September 2009.

[9] F. H. Fisher and V. P. Simmons, "Sound absorption in sea water," The Journal of the Acoustical Society of America, vol. 62, no. 3, pp. 558$564,1977$.

[10] R. J. Urick, Principles of Underwater Sound. Los Altos, California: Peninsula Publishing, third ed., 1983.

[11] A. Sehgal, I. Tumar, and J. Schönwälder, "Variability of available capacity due to the effects of depth and temperature in the underwater acoustic communication channel,' in Proc. of IEEE OCEANS '09, (Bremen), May 2009.

[12] H. Kwon and T. Birdsall, "Channel capacity in bits per joule," IEEE Journal of Oceanic Engineering, vol. 11, no. 1, pp. 97-99, 1986.

[13] H. A. Leinhos, "Capacity calculations for rapidly fading communications," IEEE Journal of Oceanic Engineering, vol. 21, no. 2, pp. 137 142, 1996.

[14] A. F. Harris and M. Zorzi, "Modeling the underwater acoustic channel in ns2," in ValueTools '07: Proceedings of the 2nd international conference on Performance evaluation methodologies and tools, (ICST, Brussels, Belgium, Belgium), pp. 1-8, ICST (Institute for Computer Sciences, Social-Informatics and Telecommunications Engineering), 2007. 